\title{
Alguns aspectos biométricos do aparelho genital externo de jumentos doadores de sêmen da raça Pêga
}

\author{
Some biometric aspects of the external genital tract from donkey Pêga breed semen donors
}

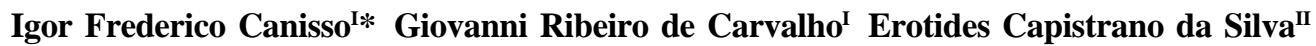 \\ Aline Luciana Rodrigues ${ }^{\text {III }}$ Pedro Game Ker ${ }^{I}$ José Domingos Guimarães ${ }^{\text {II }}$
}

\section{RESUMO}

O presente estudo foi conduzido com seis jumentos reprodutores da raça Pêga, com peso médio de $272 \pm 34,9$ (231-326kg), sendo subdivididos em adultos (J1, J2 e J3, média 15 anos) e jovens (J4, J5 e J6, média 3,5 anos). Os reprodutores apresentavam histórico normal de fertilidade à monta natural $e$, após realização de exame andrológico, foram classificados como aptos à reprodução. Foram realizadas 180 coletas de sêmen. Os reprodutores apresentaram parâmetros seminais considerados normais para a espécie. De agosto/2006 a fevereiro/2007, após a realização do exame andrológico, cada reprodutor foi submetido a três ou quatro mensurações do aparelho genital externo para: comprimento testicular (CT crânio caudal), altura testicular (AT - dorso ventral), largura testicular (LT - médio lateral) e espessura do funículo (FUN terço médio). Os dados de biometria testicular foram utilizados para o cálculo do índice testicular (IT) e volume testicular (VT). O IT médio observado foi de 7,4 (5,9 a 9,6), e o VT esquerdo foi de $155,5 \pm 14,4 \mathrm{~mL}$, enquanto o direito foi de $149,3 \pm 14,9$. A medida de FUND foi de 25,3 $\pm 3,3 \mathrm{~mm}(20,3 a$

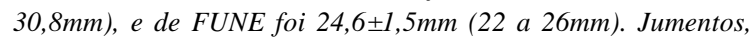
mesmo com IT menor do que o proposto para garanhões; se apresentaram mais eficientes que os últimos para a produção espermática. O volume espermático, associado ao IT, pode ser usado como ferramenta auxiliar na estimativa da constatação de normalidade para testículos de jumentos. A mensuração in vivo do funículo espermático pode ser uma nova variante na utilização do exame andrológico de jumentos. Os dados descritos podem servir de valores de referência e valores práticos quanto à biometria do aparelho genital externo de jumentos.

Palavras-chave: biometria, volume, índice testicular.

\begin{abstract}
The present study was performed with six Pêga breed donkeys, weight from 231 to $326 \mathrm{~kg}(272 \pm 34.9)$. The breeders were divided into the adult group (J1, J2 e J3, 15 years old average) and the young group (J4, J5 e J6, 3.5 years old average). . The donkeys presented normal rates of fertility at natural mating. After breeding soundness evaluation all animals were considered capable for reproduction. A hundred eight semen collections were realized, and the donkeys presented normal seminal parameters for the specie. From August/2006 to February/2007, after semen collection each donkey was submitted to three or four measurements of the extern genital tract (both right and left side) for: testis length (LT - cranio caudal); testis height (TH - dorso ventral) and thickness of spermatic funiculum (FUN - third part). The biometric data from biometric testis were used to calculate the testis indices (TI) and testis volume (TV). The TI observed was 7.4 (5.9 a 9.6) and TV was $155.5 \pm 14.4,149.3 \pm 14.9$ respectively left and right. The average FUN was $25.3 \pm 3.3 \mathrm{~mm}(20.3 \mathrm{a} 30.8 \mathrm{~mm})$ to the right and $24.6 \pm 1.5 \mathrm{~mm}$ (22 a 26mm) to the left side. The donkeys presented lesser TI than those proposed for the stallions, but the asinine were more efficient for sperm production than the average reported to stallions. The testis volume associated with IT can be used as an additional tool to estimate the normality of the breeder's testis. The measurement in vivo of spermatic funiculum can be used as a new component for the breeding soundness evaluation of donkeys. The genital extern tract biometric values can support routine breeding evaluation and can be applied to news studies.
\end{abstract}

Key words: biometric, volume, testicular indices.

ISetor de Equideocultura, Departamento de Zootecnia, Universidade Federal de Viçosa (UFV), 36570-000, Viçosa, MG, Brasil. Email: ifc@cornell.edu. *Autor para correspondência. Instituição atual: Section of Theriogenology, Hospital for Animals/Equine Research Park, Department of Clinical Sciences, College of Veterinary Medicine, Cornell University, Ithaca, NY, United States. "Departamento de Veterinária, UFV, Viçosa, MG, Brasil.

"IEscola de Veterinária, Universidade Federal de Goiás (UFG), Goiânia, Goiás, Brasil. 


\section{INTRODUÇÃO}

No Brasil, o principal interesse em jumentos se resume na doação de sêmen para a produção de muares, embora exista no país plantéis asininos de elevado padrão genético, em que esse reprodutor é usado para a produção da própria espécie. A raça Pêga é uma raça asinina nacional criada em quase todos os Estados da federação, sendo largamente usada em cruzamentos com éguas puras e mestiças para produção de muares marchadores (NUNES, 2007), necessitando, dessa forma, de avaliação precisa da qualidade seminal e do aparelho reprodutivo para possível predição da fertilidade e normalidade andrológica. Destaca-se, nesse ponto, o exame andrológico por ser importante ferramenta auxiliar de predição da capacidade reprodutiva do macho, sendo de relevância para a aplicação de biotecnologias do sêmen, na rotina dos haras, bem como em pesquisas (LOVE, 2007), auxiliando na melhoria da eficiência reprodutiva (KENNEY et al., 1983).

No Brasil, as normas e os padrões da avaliação andrológica e do sêmen foram estabelecidos pelo Colégio Brasileiro de Reprodução Animal (CBRA, 1998). Porém, o reprodutor asinino não foi incluído nos padrões estabelecidos por essa entidade. Desse modo, experimentos e levantamentos de campo devem ser realizados para sejam estabelecidos os padrões de exame andrológico para essa espécie (CANISSO et al., 2008a). A biometria testicular deve ser um requisito obrigatório nessa avaliação, uma vez que fornece informações úteis em termos de comparação do tamanho normal e, principalmente, previsão quanto à produção espermática (KENNEY et al., 1983).

A partir das biometrias testiculares, podem ser realizados cálculos de índice e volume testicular. O índice testicular pode ser utilizado como ferramenta no estabelecimento da condição de normalidade reprodutiva do órgão e como auxiliar na estimativa da produção espermática diária e assim auxiliar na predição do número de éguas e ou jumentas a serem cobertas e/ ou inseminadas com sêmen de um dado reprodutor durante a estação de monta (MORAIS, 1990). O objetivo deste estudo foi avaliar alguns aspectos da biometria do aparelho genital externo de jumentos reprodutores da raça Pêga considerados aptos à reprodução pelo exame andrológico.

\section{MATERIAL E MÉTODOS}

O presente experimento foi desenvolvido durante o período de agosto/2006 a fevereiro/2007. Foram utilizados seis reprodutores da raça Pêga, com peso médio de 272×34,9 (231-326kg) e idade média de 109 meses, no início do experimento. Os animais pertenciam a um haras de criação comercial de asininos e muares, localizado no Município de Guaraciaba, Zona da Mata de Minas Gerais. Foram subdivididos em dois grupos: adultos (J1, J2 e J3 16,14 e 16 respectivamente) e jovens (J4, J5 e J6 todos com média de 3,5 anos). Todos os reprodutores apresentavam histórico de boa fertilidade à monta natural com éguas em estações de monta anteriores.

Os jumentos foram mantidos em baias de alvenaria individuais com acesso a bebedouro, sal mineralizado comercial específico para equídeos, além de feno de gramínea (Cynodon dactylon da cultivar 'Tifton 85'), com acesso irrestrito durante todo o período do dia. Foram oferecidos $20 \mathrm{~kg}_{\text {animal }}{ }^{-1}$ de capim fresco picado (Pennisetum purpureum da cultivar 'Elefante'), divididos em dois tratos: um pela manhã e outro pela tarde. Além disso, pela manhã, antes do primeiro trato, foi fornecida diariamente a todos os reprodutores uma dose de $20 \mathrm{~mL}$ de um suplemento líquido polivitamínico e microminerais para equídeos, de acordo com a recomendação do fabricante.

Antes do início dos procedimentos, realizouse exame clínico andrológico de acordo com as recomendações de KENNEY et al. (1983) para sêmen de garanhões e de CANISSO et al. (2008a), em revisão sobre exame andrológico em asininos; sendo todos os animais classificados como aptos à reprodução. Durante o período decorrido entre as mensurações, foram realizadas 180 coletas de sêmen para avaliação dos parâmetros seminais e constatação da normalidade andrológica. Para as coletas, se fez uso de uma vagina artificial modelo Botucatu; fazendo a coleta com uso de égua em estro como descrito por CANISSO et al. (2008b, 2010). Imediatamente após a coleta, foram feitas avaliações dos parâmetros físicos (volume, motilidade, motilidade progressiva, vigor, concentração) e morfológicos do sêmen, de acordo com a metodologia de KENNEY et al. (1983).

Ao longo do período experimental, cada reprodutor foi submetido a três ou quatro mensurações, em que cada órgão foi mensurado individualmente, utilizando-se um paquímetro, conforme descrito por GEBAUER et al. (1974) e adaptado por GEBER (1995) para asininos, após a coleta de sêmen. Foram mensurados o comprimento testicular (CT - crânio caudal), a altura testicular (AT - dorso ventral) e a largura testicular (LT - médio lateral) em ambos os testículos. Além disso, realizou-se a medição da espessura do funículo (FUN - terço médio) em ambos os antimeros corporais, em cada mensuração. Foram 
feitas várias medidas sequenciais até a coincidência de três resultados iguais de cada item, para só então ser considerado o resultado válido.

As mensurações testiculares foram utilizadas para se obter o “índice testicular”, como preconizado por KENNEY (1989) apud MORAIS (1990) de acordo com a fórmula: IT= (CTE x LTE x ATE $)+$ (CTD x LTD x ATD)/100, em que: IT = índice testicular; $\mathrm{CTE}=$ comprimento testicular esquerdo; $\mathrm{LTE}=$ largura testicular esquerda; ATE = altura testicular esquerda; CTD = comprimento testicular direito; LTD = largura testicular direita; e ATD = altura testicular direita. Foi realizada a estimativa do volume testicular $(\mathrm{cm})$ empregando a fórmula preconizada por EL WISHY (1974) para testículos de jumentos e garanhões de acordo com a fórmula: $\mathrm{Y}_{\mathrm{vt}}=* 33,57 \mathrm{X}-56,57$, sendo $\mathrm{Y}_{\mathrm{vt}}$ igual ao volume testicular; " $\mathrm{X}$ " representa a altura testicular (cm); e os números 33,57 e 56,57 representam os fatores de correção constantes.

Para as análises, empregou-se o programa estatístico SAEG 9,1 (SAEG -UFV, 2007). Foram efetuados cálculos da média aritmética, do desvio padrão e do coeficiente de variação dos resultados obtidos para cada variável avaliada, por reprodutor e para o grupo experimental. Para as variáveis qualitativas, efetuaram-se os testes de Lillinfors e Cochan e Bartllet para verificar a normalidade dos dados e homogenicidade das variâncias dos grupos, nessa ordem. Os dados que não atenderam a premissa da ANOVA foram submetidos à análise não paramétrica, e as médias foram comparadas pelos testes de KrussKall Wallis ou Wilcox, com probabilidade de erro de 5\%. As variáveis que atenderam a pelo menos uma premissa foram submetidas à análise de variância por ANOVA, e as médias foram comparadas pelo teste de Tukey, com $5 \%$ de probabilidade de erro. Foram realizados testes de coeficiente de correlação de Pearson entre as variáveis.

\section{RESULTADOS E DISCUSSÃO}

Os valores médios registrados dos principais parâmetros seminais estão sumarizados na tabela 1. Os valores registrados para os parâmetros físicos do sêmen e morfológicos estiveram incluídos dentro da faixa considerada normal por outros estudos com jumentos da raça Pêga (MORAIS, 1990; COSTA, 1991; GEBERS, 1995; GASTAL et al., 1996).

Os valores de biometria testicular para ambos os órgãos encontram-se sumarizados na tabela 2. Foram diferentes entre os dois grupos $(\mathrm{P}<0,05)$. Provavelmente, porque os animais jovens (idade média
36 meses) ainda estavam em fase de desenvolvimento corporal e testicular. Gebers (1995) registrou que CTD apresenta correlação alta e positiva com emissão diária de espermatozoides $(r=0,80)$, enquanto que no presente estudo a correlação foi positiva e baixa $(r=0,26)$. Foi registrada por COSTA(1991), assim como neste estudo, relação linear positiva $(r=0,68)$ entre peso corporal e CTD. Porém, não houve diferenças no peso médio entre os grupos $(\mathrm{P}>0,05)$. O CTE, para o grupo de adultos, foi superior da média do grupo de jovens $(\mathrm{P}<0,05)$. A CTE e CTD médias foram semelhantes ( $\mathrm{P}>0,05)$.

Os valores da ATD foram superiores para o grupo dos animais jovens $(\mathrm{P}<0,05)$, provavelmente ATD se desenvolva mais precocemente que as demais características testiculares quando o órgão estiver em fase de crescimento. O valor médio de LTD não apresentou diferenças entre os grupos $(\mathrm{P}>0,05)$. $\mathrm{O}$ jumento J6 apresentou elevado LTD, o que aumentou a média do grupo de jovens. Contudo, esse parâmetro é o menos influenciável por erros de metodologia, uma vez que a mensuração é médio-lateral e não envolve o epidídimo; porém, apesar de os experimentos anteriores terem empregados a mesma raça, as condições de criação e manejo foram diferentes, o que pode ter interferido no desenvolvimento testicular dos reprodutores. É importante ressaltar que, no presente experimento, teve-se o cuidado de realizar a mensuração, empregando-se a contenção dos reprodutores em tronco específico para equídeos. Entretanto, os demais parâmetros e as médias para ATE e LTE foram maiores, porém não diferentes para adultos em relação aos jovens ( $\mathrm{P}>0,05)$. Isso, provavelmente, devido ao fato de os adultos terem completado o desenvolvimento testicular em oposição aos demais que ainda estavam em fase de desenvolvimento.

Tabela 1 - Valores médios \pm desvio padrão de parâmetros seminais de seis asininos doadores de sêmen da raça Pêga (agosto/2006 - fevereiro/2007).

\begin{tabular}{ll}
\hline Parâmetro & \multicolumn{1}{c}{ Mensuração } \\
\hline Volume Seminal livre de gel & $47,2 \mathrm{~mL} \pm 28,6 \mathrm{~mL}$ \\
Volume e ${ }^{\circ}$ de ejaculados & $71,7 \mathrm{~mL} \pm 54,8 \mathrm{~mL}$ \\
com gel & $(54 / 180$ coletas $)$ \\
Motilidade total & $84,2 \% \pm 6,0$ \\
Motilidade progressiva & $74,4 \% \pm 7,0$ \\
Vigor espermático (1-5) & $3,8 \pm 0,5$ \\
Defeitos espermáticos maiores & $7,9 \% \pm 3,0 \%$ \\
Defeitos espermáticos & $6,8 \pm 1,5 \%$ \\
menores & $25,6 \times 10^{6} \mathrm{~mL}^{-1}$ \\
Concentração espermática & $10,3 \times 10^{9} \pm 452,5$ \\
Numero de espermatozoides & $\left(6,3\right.$ a $\left.12,5 \times 10^{9}\right)$ \\
totais no ejaculado & \\
\hline
\end{tabular}


Alguns aspectos biométricos do aparelho genital externo de jumentos doadores de sêmen da raça Pêga.

Tabela 2 - Médias e desvio padrão (mm) das biometrias do testículo direito e esquerdo (comprimento, altura e largura) de seis reprodutores asininos adultos e jovens da raça Pêga.

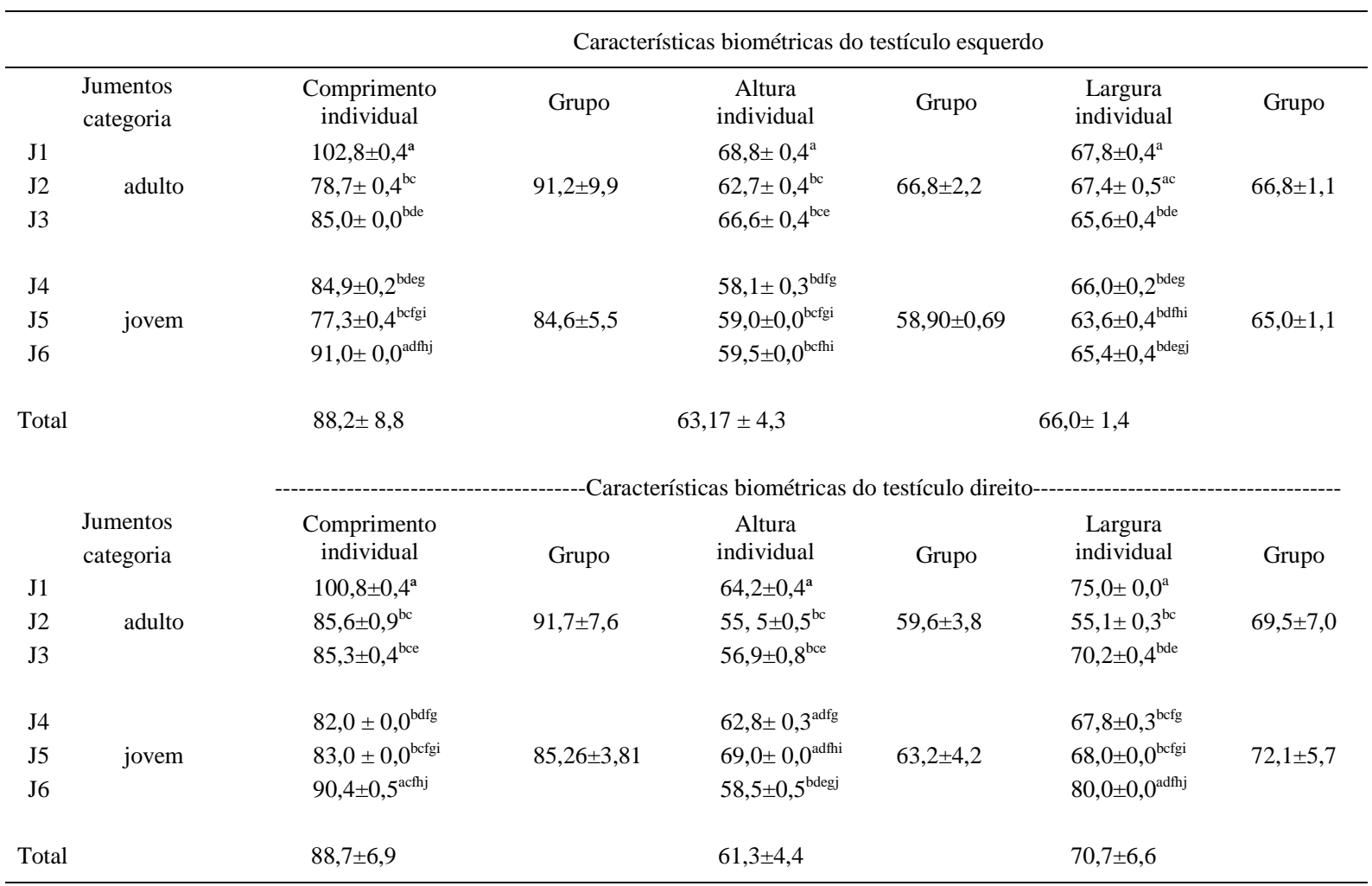

*Médias e Desvio Padrão seguidas por letras minúsculas diferentes na mesma coluna diferem entre si $(\mathrm{P}<0,05)$ para o Teste de KruskalWallis.

Os valores registrados para o índice testicular encontram-se sumarizados na tabela 3. O índice testicular (IT) médio observado foi de 7,45, e as variações ficaram entre 5,94 e 9,64, sendo inferiores ao mínimo (IT=8) preconizado para garanhões por Kenney (1989) apud MORAIS (1990). No entanto, todos os reprodutores apresentavam histórico reprodutivo de boa fertilidade em pelo menos uma estação reprodutiva, e os parâmetros seminais se apresentaram na faixa considerada normal para asininos.

Valores superiores ao presente estudo foram registrados por MORAIS (1990) em seis reprodutores asininos, com valores de IT de 8,24 a 12,73. Calculandose o IT a partir dos dados mensurados por COSTA (1991) e GEBERS (1995), foram obtidos os valores de 7,90 e 8,12 para IT em jumentos Pêga, andrologicamente normais. Provavelmente, as diferenças de tamanho corporal, bem como as condições de criação dos reprodutores, possam ter sido responsáveis pelas diferenças nas características de biometria testicular e, consequentemente, no IT.

O valor referido por Kenney (1989) apud MORAIS (1990) para garanhões, quando aplicado a jumentos, deve ser reconsiderado, de modo que se constata que o valor de 8,0 é muito alto para a espécie em questão, em que neste estudo, apesar de valores de 7,45 de IT, os animais apresentaram-se normais quanto à eficiência na produção espermática, manifestada pelo número de espermatozoides totais (Tabela 1), presentes no ejaculado dentro da faixa considerada normal para a espécie (KREUCHAUF, 1984; MORAIS, 1990, COSTA, 1991, GEBERS, 1995), e superior aos registrados para garanhões (KENNEY et al. 1983). Entretanto, no presente estudo, o valor de IT foi semelhante entre os grupos $(\mathrm{P}>0,05)$

Os jumentos, mesmo apresentando IT menor, possuem entre todos os animais domésticos a maior eficiência espermatogênica por grama de parênquima testicular (NEVES, 2001). Assim, novos experimentos devem ser conduzidos para ser possível estabelecer o valor ideal para reprodutores asinino doadores de sêmen.

Os valores registrados para o volume testicular encontram-se sumarizados na tabela 3. Não foram observadas diferenças nas comparações de VTE e VTD para um mesmo animal $(\mathrm{P}<0,05)$. Ao comparar 
Tabela 3 - Médias e desvio padrão (mm) do índice testicular e volume testicular esquerdo e direito, obtidos a partir de mensurações testiculares de seis reprodutores da raça Pêga.*

\begin{tabular}{|c|c|c|c|c|}
\hline \multicolumn{2}{|c|}{$\begin{array}{l}\text { Jumentos } \\
\text { Categoria }\end{array}$} & Índice testicular (IT) & Volume testicular esquerdo (VTE) & Volume testicular direito (VTD) \\
\hline $\mathrm{J} 1$ & & 9,6 & $174,3 \pm 1,3^{\mathrm{a}}$ & $158,9 \pm 1,3^{\mathrm{a}}$ \\
\hline $\mathrm{J} 2$ & Adulto & 5,9 & $153,9 \pm 1,5^{\mathrm{bc}}$ & $130,0 \pm 1,7^{\mathrm{bc}}$ \\
\hline J3 & & 7,1 & $167,0 \pm 1,6^{\text {bcd }}$ & $134,4 \pm 2,7^{\text {ace }}$ \\
\hline J4 & & 6,7 & $138,6 \pm 1,1^{\text {bcef }}$ & $154,4 \pm 1,1^{\text {adfg }}$ \\
\hline J5 & Jovem & 6,7 & $141,4 \pm 0,3^{\text {bcefh }}$ & $175,0 \pm 0,0^{\text {adfhi }}$ \\
\hline J6 & & 7,7 & $143,3 \pm 1,6^{\text {bcefh }}$ & $139,9 \pm 1,6^{\text {adeg }}$ \\
\hline & & 7,4 & $155,5 \pm 14,4$ & $149,3 \pm 14,9$ \\
\hline
\end{tabular}

*Médias e desvio padrão seguidos por letras minúsculas diferentes na mesma coluna diferem entre si $(\mathrm{P}<0,05)$ para o Teste de KruskalWallis. IT: índice testicular; VT; volume testicular.

estudos com jumentos da raça Pêga, envolvendo o volume testicular, reportado por MORAIS (1990) (VTE: 201,36 $\pm 36,38 m L$ e VTD:182,34 $\pm 32,58 m L\{n=6\})$; e a partir do cálculo aproximado do volume testicular com uso dos valores biometricos de animais da mesma raça reportados por COSTA(1991)(VTE: 174,78mL e VTD: 181,49 mL $\{n=103\})$, e por GEBERS (1995)(VTE: 184,51 e VTD: $181,15 \mathrm{~mL}\{\mathrm{n}=6\}$ ) com o presente estudo, os dados aqui se mostrarão ligeiramente inferiores, porém superiores aos reportados para a raça Nordestina (volume testicular médio 127,45mL; GASTAL, 1991). Isso se deve provavelmente a diferenças de tamanho corporal entre os estudos. A mensuração do volume testicular pode ser uma ferramenta útil na avaliação andrológica e na predição da produção espermática, uma vez que propicia dimensões globais do órgão e facilitaria a comparação entre animais sob condições variadas de criação. Na comparação dos resultados das características de biometria testicular de jumentos da raça Pêga do presente experimento com as demais raças, observa-se que estes, por serem animais de médio-grande porte dentro da espécie, apresentam características de biometria testicular superiores àquelas relatadas para jumentos da raça Nordestina por GASTAL (1991) e para animais mestiços de origem africana relatados por KREUCHAUF (1984).

O peso médio relatado pelos autores nesses dois experimentos foi de $162 \mathrm{~kg}$ e $150 \mathrm{~kg}$, enquanto que, nesta pesquisa, a média foi de $272 \mathrm{~kg}$. Em uma comparação das características do presente experimento com outros autores (MORAIS, (1990) e GEBERS, (1995) que realizaram biometria testicular em jumentos da raça Pêga, observou-se diferença média de até $130 \mathrm{~kg}$, a qual pode ser explicada pela inferioridade em quase todos os parâmetros de biometria testicular avaliados neste experimento em relação aos estudos com jumentos da raça Pêga (MORAIS, 1990; COSTA, 1991, GEBERS, 1995) e pela superioridade dos resultados deste em relação aos demais, em que foram utilizadas raça e animais de menor porte em relação ao jumento Pêga (GASTAL, 1991).

Os valores médios registrados para a largura do terço médio do funículo espermático direito e esquerdo encontram-se sumarizados na tabela 4 . O valor médio da largura do terço médio do funículo espermático direito e esquerdo foi semelhante ao valor preconizado como normal para garanhões por KENNEY et al. (1983). Não foram verificados estudos na literatura consultada envolvendo as dimensões do funículo espermático de jumentos.. Essa estrutura se faz relevante, pois é por ela que são conduzidos os nutrientes ao testículo para que seja possível a realização da espermatogênese, o transporte de hormônio masculino e também o mecanismo de resfriamento do sangue arterial (THOMPSON, 1992).

NORONHA et al. (2001) realizaram estudo da morfologia do funículo espermático de jumentos da raça Pêga e registraram diferenças em relação a todas as demais espécies domésticas e silvestres estudadas, sendo caracterizada no estudo a presença de um tecido subcapsular entremeado com tecido muscular que pode ser responsável pela facilitação do retorno venoso do testículo e, consequentemente, maior eficiência no processo de drenagem, facilitando a espermatogênese. Ao realizar-se a avaliação entre jovens e adultos, os valores para FUNE foram semelhantes $(\mathrm{P}>0,05)$ (Tabela 4), em oposição ao órgão direito (FUND), em que foram observadas diferenças entre os grupos de adultos e jovens $(\mathrm{P}<0,05)$, e o primeiro grupo apresentou valor superior ao segundo grupo, provavelmente por estarem em processo de desenvolvimento corporal e testicular.

Ciência Rural, v.39, n.9, dez, 2009. 
Tabela 4 - Médias e desvio padrão (mm) da largura do terço médio do funículo espermático direito e esquerdo, de seis reprodutores da raça Pêga.*

\begin{tabular}{|c|c|c|c|c|c|}
\hline \multirow{3}{*}{\multicolumn{2}{|c|}{$\begin{array}{l}\text { Jumentos } \\
\text { Categoria }\end{array}$}} & \multirow{2}{*}{\multicolumn{2}{|c|}{---Funículo }} & \multicolumn{2}{|c|}{ 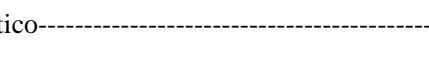 } \\
\hline & & & & \multirow{2}{*}{ Geral } & \multirow{2}{*}{ Categoria } \\
\hline & & \multirow{2}{*}{$\frac{\text { Geral }}{30,8 \pm 0,4^{\mathrm{a}}}$} & Categoria & & \\
\hline $\mathrm{J} 1$ & \multirow{3}{*}{ Adulto } & & \multirow{3}{*}{$27,5 \pm 2,9$} & $26,0 \pm 0,0^{\mathrm{a}}$ & \multirow{3}{*}{$24,4 \pm 1,3$} \\
\hline $\mathrm{J} 2$ & & $23,4 \pm 0,5^{\mathrm{bc}}$ & & $23,0 \pm 0,0^{\mathrm{bc}}$ & \\
\hline J3 & & $26,0 \pm 0,0^{\text {bde }}$ & & $23,6 \pm 0,4^{\text {bce }}$ & \\
\hline $\mathrm{J} 4$ & \multirow{3}{*}{ Jovem } & $23,8 \pm 0,3^{\text {bcfg }}$ & \multirow{3}{*}{$22,8 \pm 1,6$} & $26,0 \pm 0,0^{\text {adfg }}$ & \multirow{3}{*}{$24,7 \pm 1,8$} \\
\hline J5 & & $20,3 \pm 0,4^{\text {bcfgi }}$ & & $22,0 \pm 0,0^{\text {bcfhi }}$ & \\
\hline J6 & & $24,0 \pm 0,0^{\text {bcfhj }}$ & & $26,0 \pm 0,0^{\text {adfgj }}$ & \\
\hline & & $25,3 \pm 3,3$ & & $24,6 \pm 1,5$ & \\
\hline
\end{tabular}

*Médias e desvio padrão seguidos por letras minúsculas diferentes na mesma coluna diferem entre si $(\mathrm{P}<0,05)$ para o Teste de KruskalWallis.

Registrou-se, no presente experimento, correlação alta e positiva entre FUND e CTD $\left(r^{2}=0,86\right)$, enquanto que, para os órgãos do lado esquerdo, a correlação foi baixa. Sugere-se que a mensuração do funículo espermático seja incluída na avaliação andrológica de jumentos, pois pode ser um parâmetro auxiliar na interpretação dos resultados do exame andrológico.

\section{CONCLUSÃO}

Os jumentos do presente estudo, mesmo com IT menor que o proposto como normal para garanhões, apresentaram-se mais eficientes que animais da espécie equina para a produção espermática. Maior número de animais deve ser avaliado para que se possa estabelecer o IT normal para asininoscriados nas condições brasileiras. O volume espermático, associado ao IT, pode ser usado como objeto auxiliar na estimativa da normalidade para testículos de jumentos. A mensuração in vivo do funículo espermático pode ser uma nova variante na utilização do exame andrológico de jumentos. Os dados aqui descritos podem servir de valores de referência e valores práticos frente à escassez de dados para a biometria do aparelho genital externo de jumentos.

\section{AGRADECIMENTOS}

Ao Conselho Nacional de Desenvolvimento Científico e Tecnológico (CNPq), pelo suporte financeiro, e ao Sr. Luis Felipe Haddad (Associação Brasileira de Criadores de Jumento Pêga), pela concessão dos animais.

\section{REFERÊNCIAS}

CANISSO, I.F. et al. Sexual behavior and ejaculate characteristics in Pêga donkey (Equus asinus) mounting estrous horse mares (Equus caballus). Theriogenology, (in press), 2010.

CANISSO, I.F. et al. Alguns aspectos fundamentais do exame clinico andrológico de jumentos (Equus asinus). Rev Bras Reprod Animal, (in press), 2008.

CANISSO, I.F. et al. Coleta de sêmen de jumentos (Equus asinus) utilizando-se eguas em estro como manequim. Cien Vet Trop, v.11, n.2/3, 2008 (in press).

CBRA (Colégio Brasileiro de Reprodução Animal). Manual para exame andrológico e avaliação de sêmen animal. 2.ed. Belo Horizonte, 1998. 54p.

COSTA, A.J.S.A. Avaliação clínico andrológica do jumento da raça Pêga. 1991. 66f. Dissertação-Mestrado em Medicina Veterinária, Escola de Veterinária - Universidade Federal de Minas Gerais, Belo Horizonte, MG.

EL WISHY, A.B. Testicular and epididymal sperm reserves in the ass (Equus asinus) and stallion (Equus caballus). $\mathbf{Z}$ Tierzuchig Zucstblol, v.91, p.344-44, 1974.

GASTAL, M.O. et al. Sexual behavior of donkey jacks: influence of ejaculatory frequency and season. Theriogenology, v.46, n.4, p.593-603, 1996. Disponível em: <http://www.sciencedirect.com/ science?_ob=ArticleURL\&_udi=B6TCM-3W30SN7$3 \&$ \& u s e r $=492137 \&$ \& c ov e r D a t e $=09 \% 2$ F $30 \% 2$ F 1996 \&_a li d = 987174724 \&_rd oc $=5 \&$ \&mt $=$ high \&_orig $=$ search\&_cdi $=5174 \&$ \&sort $=$ r\&_docanch or $=\&$ vi e w $=$ c \&_ct $=16 \&$ \& a c ct $=$ C $000022719 \&$ _ version $=1 \&$ \& u r l Versio $n=0 \&$ _ us e r i d $=49$ 2137\&md5=1a08a9cc68b236d124a2debae3cbadf9>. Acesso em: 5 mar. 2008. doi:10.1016/0093-691X(96)00211-7.

GASTAL, M.M.F.O. Estudo das características seminais e do comportamento sexual de jumentos. 1991. $105 \mathrm{f}$. Dissertação - Mestrado em Medicina Veterinária, Escola de 
Veterinária - Universidade Federal de Minas Gerais, Belo Horizonte, MG.

GEBAUER, M.R. et al. Reproductive physiology of the stallion: Daily sperm output and testicular measurements. Journal Amer Vet Med Assoc, v.165, n.8, p.711-13, 1974.

GEBERS, A.M. Emissão diária de espermatozóides e algumas características reprodutivas de jumentos da raça Pêga. 1995. 90f. Dissertação (Mestrado em Zootecnia) - Departamento de Zootecnia, Universidade Federal de Viçosa, MG.

KENNEY, R.M. et al. Manual for clinical fertility evaluation of the stallion. Hastings: Journal Society Theriogenology, v.9, 100p. 1983.

KREUCHAUF, A. Reproductive physiology in the jackass. Animal Research Development, v.20, p.51-78, 1984.

LOVE, C.C. Reproductive examination of stallion: evaluation of potential breeding soundness. In: YOUNGQUIST, R.S.; THREFALL, W.R. Current therapy in large animal theriogenology. 2.ed. Saint Louis: Saunders-Elsevier, 2007. p.10-14.

MORAIS, R.N. Contribuição ao estudo da biologia reprodutiva de jumentos (Equus asinus). 1990. $105 f$.
Dissertação (Mestrado em Medicina Veterinaria) - Faculdade de Medicina Veterinária e Zootecnia, Universidade de São Paulo, SP.

NEVES, E.S. Estudo comparativo da estrutura do testículo e do processo espermatogênico em jumentos (Equus asinus) e burros (Equus mulus mulus). 2001. 135f. Tese (Doutorado em Ciencias Biologicas) - Instituto de Ciências Biológicas, Universidade Federal de Minas Gerais, Belo Horizonte, MG.

NORONHA, P.B. et al. Aspectos morfológicos do funículo espermático de jumentos (Equus asinus Linnaeus, 1758) da raça Pêga. Braz J Vet Res Anim Sci, v.38, n.5, p.209213, 2001. Disponível em: <http://www.scielo.br/ scielo.php? script=sci_arttext\&pid=S 1413 $95962001000500002 \& \mathrm{t} \operatorname{lng}=\mathrm{en} \& \operatorname{lng}=\mathrm{en} \& \mathrm{nrm}=\mathrm{i}$ so $>$. Acesso: 5 mar. $2008 . \quad$ doi: 10.1590/S141395962001000500002 .

NUNES, R. O Jumento Pêga. In: CARVALHO, G.R. et al. SIMPÓSIO MINEIRO DE EQÜIDEOCULTURA, 2007, Viçosa, MG. Anais... Viçosa: Universidade Federal de Viçosa, 2007. V.1, n.1, p.20-31, 2007.

THOMPSON, D.L. Reproductive physiology of stallion and jack. In: EVANS, J.W. World animal science: horse breeding and management. London: Elsevier, 1992. p.237257. 